\section{Training of French Hospital Personnel during CBRN Emergencies}

Catberine Bertrand; ${ }^{1}$ Eric Lecarpentier; ${ }^{2}$ Alain Margenet ${ }^{2}$

1. Training of French Hospital Personnel in NRBC Decontamination

2. SAMU 94 Samu de France

In France, the hospital health plan called the White Plan contains specific sections to cope with chemical, biological, radiological, or nuclear risks (CBRN). Training personnel and organizing exercises within the plan are mandatory. This training began in 2002 after the conception of a civilmilitary doctrine by a national steering committee. Fourday training seminars occurred at a national then zonal level, and were divided into two-day seminars for the additional teachers. Since 2002, these additional teachers trained $>30,000$ people from emergency services in oneday seminars. Since 2006, additional effort has been made under the responsibility of the emergency teaching centre. First, voluntary health professionals who also are medically fit are chosen to run a decontamination area at the entrance of their hospitals. These people are specifically trained for nine hours and receive a diploma that is valid for two years. Second, anybody working in a hospital must be trained every four years in emergency procedures including general information on CBRN risks. These subjects must be taught in a hands-on manner with the objective of changing traditional health professionals' perceptions. Finally, training is a continuing learning and adapting process.

Keywords: chemical, biological, radiological, or nuclear; education;

France; hospital personnel; training

Prebosp Disaster Med

\section{Promoting Hospital Preparedness to Chemical Events} through Exercises

A. Abramovich; ${ }^{1}$ A. Obana, ${ }^{1}$ R. Coben; ${ }^{2,3,4}$ B. Adini;

B. Azaria

1. Hospital Preparedness Branch, Home Front Command, Israel Defense Forces, Israel

2. Ministry of Health, Israel

3. Faculty of Emergency Medicine and University Research Institute of Disaster Readiness and Preparedness, Ben Gurion University, Israel

4. The National Institute for Trauma Research and Emergency Medicine, Gertner Institute, Israel

Background: Non-conventional war due to exposure to chemical warfare agents is one of the emergency scenarios that require readiness and preparedness. In order to promote preparedness, hospitals undergo drills and exercises. The drills are complex exercises, requiring significant resources (financial costs, time, personnel, and equipment). Methods: During hospital exercises performed during 2005-2008, a questionnaire was used to assess and inspect the exercises. The questionnaire assisted in performing supervision of the different sites and units that operate in the hospital, which are being evaluated during the exercise. The questionnaire is completed by inspectors from another hospital, which recently has undergone a similar exercise. Results: The average scores sites in the sites before the yellow line was $85 \pm 9.2 \mathrm{SD}$. The average scores at the treatment sites after the yellow line was $91 \pm 1.76 \mathrm{SD}$. The average scores for the operation and management units in the hospital was $87 \pm 1.78 \mathrm{SD}$. The difference between the average scores in the hospital management and operation units against the average scores of sites before the yellow line, was show to be statistically significant $(p=0.043)$. No other statistically significant differences between the other sites were demonstrated.

Conclusions: The difference between the sites before the yellow line and the operational sites in the hospital may be due to a combination of factors that include the following: non-organic hospital staff, non-skilled and under-trained staff that perform fundamentally different work during the layout task from the day-to-day work, and finally, using a protective garment, makes functioning and communication more difficult.

Using the tool facilitates the reception of data and creates a snapshot, which enables a global vision of the functioning of the hospital in the event in the different treatment sites. This overview facilitates focusing on the weak points of the hospital. Hence, the results assist in learning lessons and drawing conclusions that enable improvement in future exercises and responses to real events.

Keywords: chemical events; drills; exercises; hospitals; preparedness Prebosp Disaster Med

\section{New Trends in Hospital Preparedness for Radiation} Incidents with Mass Casualties

\section{Tal Brosh Nissimov;, Ido Layish; ${ }^{1}$ Chen Shapira ${ }^{2}$}

1. CBRN medicine branch, Medical corps, Israel Defense Force, Israel

2. Carmel Hospital, Haifa, and the Advising Committee for the Preparedness of Hospitals to Radiation Events, Ministry of Health, Israel

There are many challenges to hospital preparedness for radiation incidents with massive numbers of casualties. Immediate medical care according to the Advanced Trauma Life Support (ATLS) should be provided to trauma victims that might be externally and internally contaminated with radioactive materials, while attention should be given to prevent contamination of the medical staff and the hospital facilities. The conservative approach, which is recommended by various authorities and is practiced in many hospitals, dictates keeping the emergency department clear of contamination. Therefore, decontamination and radiation surveys must be conducted outside of the emergency department. This approach might lead to dangerous delays in medical treatment and might not prevent the contamination of the hospital facilities. Discussion of the disadvantages of this approach will be presented; a more liberal approach will be discussed, in which radiation casualties are admitted to the emergency department immediately upon their arrival, and are decontaminated and surveyed on site along with the provision of medical care. In this approach, the emergency room is contaminated to some degree, while the rest of the hospital remains clean, and the patients are being treated sooner and better. The concepts are based on the authors' vast experience in large hospital drills and collaboration with hospital staffs and the Ministry of Health of Israel.

Keywords: contamination; hospitals; mass-casualty incidents; preparedness; radiation

Prebasp Disaster Med 